\title{
Multivariate Approaches in Neuroimaging: Assessing the Connectome of Alzheimer's Disease
}

\author{
Juan Manuel Górriz ${ }^{\mathrm{a}, \mathrm{b}, *}$, Eugenio Iglesias-González ${ }^{\mathrm{c}}$ and Javier Ramirez ${ }^{\mathrm{a}}$ \\ ${ }^{a}$ University of Granada, Spain \\ ${ }^{\mathrm{b}}$ University of Cambridge, $U K$ \\ ${ }^{\mathrm{c}}$ University College London, UK
}

The increasing spread of in vivo imaging technologies, such as magnetic resonance imaging (MRI), diffusion tensor imaging, functional MRI, single photon emission computed tomography, positron emission tomography (PET), and of other noninvasive techniques that record electrical activity, such as electroencephalography (EEG) or magnetoencephalography, have meant a breakthrough in the diagnosis of neurodegenerative diseases, such as Alzheimer's disease (AD).

The aim of this mini-forum in the Journal of Alzheimer's Disease is to present the current stateof-the-art in the theory of multivariate approaches to assess the evolution of neurodegeneration in $\mathrm{AD}$, and to provide a quantitative prediction of changes in connectivity as disease progresses. The papers in this issue focus on exploring changes in brain connectivity caused by neurodegenerative disorders such as $\mathrm{AD}$; prediction or evolution from mild cognitive impairment (MCI) to $\mathrm{AD}$; and characterization of $\mathrm{AD}$ by multivariate approaches. In this sense, the fields of research covered in the issue are vast: from a theoretical point of view [1-4] by proposing models for neurodegeneration; at the clinical level [5-7] by assessing novel treatments, image acquisition protocols, and genetic biomarkers; to the recent advances in machine learning for the diagnosis and prognosis of $\mathrm{AD}[8-11]$.

\footnotetext{
${ }^{*}$ Correspondence to: Prof. Juan Manuel Górriz, E-mail: gorriz@ugr.es.
}

This mini-forum presents recent advances on structural and functional brain connectivity in $\mathrm{AD}$ neurodegeneration. Most of the approaches for brain image analysis are based on univariate methods. This kind of analysis is performed using one single feature at the time. They frequently consist of voxelwise comparisons of either tissue probability maps (voxel-based morphometry) or measures derived from image registration-typically the Jacobian determinant (tensor-based morphometry). However, there exists an increasing interest in multivariate approaches. These, in contrast to univariate analysis, can effectively handle patterns that arise from different brain areas simultaneously, as well as characterize the relationship between different regions of interest (ROIs). Additionally, another solution is to perform a reduced number of discriminative features, such as eigenbrain-based methods, multivariate Gaussian methods, codebook-based methods, or support vector machine-based methods.

A crucial aspect of $\mathrm{AD}$ is the disruption of brain connectivity. While neuroimaging enables the estimation of structural and functional connectivity across the brain, the connectivity matrices describing $\mathrm{AD}$ and healthy aging are still poorly understood, as is their evolution in the course of the disease. Among multivariate approaches, dynamic causal modeling is a framework that can be used for making inferences about changes in brain connectivity using neuroimaging data, while considering their causal architecture. In [1], dynamic causal models are fitted 
to high-density EEG data from subjects performing a semantic picture matching task. This work answers several important issues such as the optimal model architecture for explaining event-related potentials, or which connections are different between groups performing the same task. In MRI, other multivariate methods are available to distinguish subjects in the prodromal stage of AD [2]. This work utilizes spherical brain mapping (SBM) for detecting differences between $\mathrm{AD}$ and aging subjects without symptoms of dementia. In addition, the SBM maps showed statistical and morphological information of the brain in a bidimensional plane, thus providing a significant feature reduction, which is useful for visualization and classification purposes. Other models for brain neurodegeneration are presented in [3], where Markov random fields are proposed to study the interregional associations and dependencies between multimodal imaging markers of $\mathrm{AD}$, and to compare different hypotheses regarding the spread of the disease. This approach relies on probabilistic graphical modeling procedures that represent the interaction between individual random variables by an undirected graph. In [4], graph topology metrics derived from a graphical model assessing fiber pathways showed preserved network organization in patients compared to controls. Using a diffusion model, the effect of network alterations on degeneration spread in $\mathrm{AD}$ is proved, independently of the choice of connectome which does not significantly impact the predictive ability of the model.

At the clinical level, biomarkers of neurodegeneration play a major role in the diagnosis of AD. A novel clinical procedure is proposed in [5] to obtain information on both amyloid- $\beta(\mathrm{A} \beta)$ accumulation (e.g., from amyloid PET) and downstream neuronal injury (e.g., from FDG-PET) using a single acquisition. The parallelism between brain perfusion and glucose metabolism allows to evaluate whether brain perfusion estimated in a dual-point protocol of FBBPET can be a surrogate of FDG, using the area under the ROC curve as criterion. This mini-forum also includes another clinical study related to the loss of body weight in late-life that is known to occur at a very early stage of $\mathrm{AD}$ [6]. As in [5], composite cortical $\left[{ }^{18} \mathrm{~F}\right]-\mathrm{AV} 45$ and $\left[{ }^{18} \mathrm{~F}\right]-\mathrm{FDG}$ uptakes are considered as surrogates of cortical $\mathrm{A} \beta$ load and glucose metabolism, respectively. The relationships between these PET biomarkers with body mass index, present cognitive performance, and cognitive changes over time, were analyzed in this study. In the same lines of reasoning, the connections with late-life depression-even when of subsyndromal severity-were longitudinally investigated with the effect of serotonin selective reuptake inhibitor medication on amyloidosis and grey matter volume in [7]. This medication has been proven to distinctly rescue the declining cognitive performance in cognitively affected patients with depressive symptoms, and likewise attenuated grey matter atrophy.

Machine learning is currently providing greater insight in the description of $\mathrm{AD}$ as an application field. Many prediction modeling and pattern recognition approaches effectively reduce the dimensions of neuroimaging biomarkers, i.e., using a multi-modality sparse representation approach. In [8], multi-modal features from automatically defined brain regions-of-interest are found to be discriminative and robust for $\mathrm{AD}$ and MCI classification from T1 and FDG-PET images. The relationship between brain structure and function is highlighted by a sparse representation of brain structures that is obtained by a feature extraction procedure as well. In [9], a feature extraction process based on the relevance of the differences found between different types of subjects in each brain ROI and a greedy classification algorithm is proposed. The sparse representations of brain data enable the application of a wide range of complex classification algorithms to achieve good classification results on segmented MRI, while improving efficiency and interpretability. Another relevant imaging modality in $\mathrm{AD}$ is magnetoencephalography, which stands out by its high temporal resolution and noninvasiveness. Within this framework, the application of Coherence and Granger causality for effective functional connectivity assessment showed a significant decrease in the connectivity values for MCI patients [10]. Finally, novel advances in signal processing including wavelet analysis, artificial neural networks and predator-prey particle swarm optimizations were proposed in [11] to provide a robust and effective computer aided diagnosis system that outperforms 10 state-of-the-art approaches, which is a great help particularly for the performance of human experts.

\section{REFERENCES}

[1] Penny W, Iglesias-Fuster J, Quiroz YT, Lopera FJ, Bobes MA (2018) Dynamic causal modeling of preclinical autosomal-dominant Alzheimer's disease. J Alzheimers Dis 65, 697-711.

[2] Martinez-Murcia FJ, Górriz JM, Ramírez J, Segovia F, Salas-Gonzalez D, Castillo-Barnes D, Ortiz A, Alzheimer's Disease Neuroimaging Initiative (2018) Assessing mild 
cognitive impairment progression using a spherical brain mapping of magnetic resonance imaging. J Alzheimers Dis 65, 713-729.

[3] Dyrba M, Grothe MJ, Mohammadi A, Binder H, Kirste T, Teipel SJ, Alzheimer's Disease Neuroimaging Initiative (2018) Comparison of different hypotheses regarding the spread of Alzheimer's disease using Markov random fields and multimodal imaging. J Alzheimers Dis 65, 731-746.

[4] Powell F, Tosun D, Sadeghi R, Weiner M, Raj A, Alzheimer's Disease Neuroimaging Initiative (2018) Preserved structural network organization mediates pathology spread in Alzheimer's disease spectrum despite loss of white matter tract integrity. $J$ Alzheimers Dis 65, 747-764.

[5] Segovia F, Gómez-Río M, Sánchez-Vaño R, Górriz JM, Ramírez J, Triviño-Ibañez E, Carnero-Pardo C, MartínezLozano MD, Sopena-Novales P (2018) Usefulness of the dual-point amyloid-PET scan in appropriate use criteria: A multicenter study. J Alzheimers Dis 65, 765-779.

[6] Blautzik J, Kotz S, Brendel M, Sauerbeck J, Vettermann F, Winter Y, Bartenstein P, Ishii K, Rominger A, Alzheimer's Disease Neuroimaging Initiative (2018) Relationship between body mass index, ApoE4 status, and PET-based amyloid and neurodegeneration markers in amyloid-positive subjects with normal cognition or mild cognitive impairment. J Alzheimers Dis 65, 781-791.
[7] Brendel M, Sauerbeck J, Greven S, Kotz S, Scheiwein F, Blautzik J, Delker A, Pogarell O, Ishii K, Bartenstein P, Rominger A, Alzheimer's Disease Neuroimaging Initiative (2018) SSRI treatment improves cognition and grey matter atrophy but not amyloid burden during two-year followup in mild cognitive impairment and Alzheimer's disease patients with depressive symptoms. J Alzheimers Dis $\mathbf{6 5}$, 793-806.

[8] Kwak K, Yun HJ, Park G, Lee JM, Alzheimer's Disease Neuroimaging Initiative (2018) Multi-modality sparse representation for Alzheimer's disease classification. J Alzheimers Dis 65, 807-817.

[9] Ruiz E, Ramírez J, Górriz JM, Casillas J (2018) Alzheimer's disease computer-aided diagnosis: histogram-based analysis of regional MRI volumes for feature selection and classification. J Alzheimers Dis 65, 819-842.

[10] Gómez C, Juan-Cruz C, Poza J, Ruiz-Gómez SJ, GomezPilar J, Núñez P, García M, Fernández A, Hornero R (2018) Alterations of effective connectivity patterns in mild cognitive impairment: An MEG study. J Alzheimers Dis 65, 843-854.

[11] Zhang Y, Wang S, Sui Y, Yang M, Liu B, Cheng H, Sun J, Jia W, Phillips P, Gorriz JM (2018) Multivariate approach for Alzheimer's disease detection using stationary wavelet entropy and predator-prey particle swarm optimization. J Alzheimers Dis 65, 855-869. 